\title{
TUBERCULIN IN OPHTHALMOLOGY
}

B Y

\author{
F. A. Williamson-Noble
}

LONDON

IT has fallen to the lot of all of us at times to have to treat obstinate chronic inflammatory conditions of the eye where no definite cause has been discoverable for the condition. I am referring to diseases such as keratitis profunda, irido-cyclitis, choroido-retinitis, and persistent fine vitreous opacities without any obvious fundus lesion.

The ordinary routine examinations are performed. A Wassermann reaction is done, the teeth are examined radiologically and clinically, the tonsils, nasopharynx and sinuses are overhauled and search is made for evidences of chronic prostatitis and vesiculitis, all with negative results; or some positive finding is made, e.g., a few infected teeth are found, and the patient shows no improvement after their extraction. Possibly the examination is carried still further, cultures are made from the stools and urine, and a barium meal may even be given to find out if there be intestinal stasis, and yet no definite cause can be discovered for the patient's condition. In such a state of affairs it has been my custom, and I am sure that of many others, to "try tuberculin," and it is surprising how often the trial proves completely successful. The object of this paper is

1. To try and give some experimental explanation for the success of this reagent, in cases which are obviously not tuberculous.

2. To give some clinical experiences.

To start with the theoretical and experimental side:-When vaccines were first introduced, there was a general idea that their effect was essentially specific and that the employment of an autogenous vaccine was all important, but it has now become apparent that there is such a thing as non-specific immunity, and farther that the non-specific response may be more potent than the specific one. To show the sort of thing that happens with tuberculin B.E., I should like to quote one of Sir Almroth Wright's numerous experiments. He took a series of capillary tubes and drew into one 10 cubic millimetres of a broth suspension of staphylococcus, then 10 separate cubic millimetres of blood. The first cubic millimetres would obviously get the heaviest dose of staphylococci and the following ones progressively less, so that he had in his tube a series of 10 separate cubic millimetres of blood implanted with a progressively diminishing number of staphylococci. This tube was used as a control and contained untreated blood; in the second the blood was inoculated with a small dose of tuberculin 
and in the third with a larger dose. Wright has already shown that in vitro the immunising response to a vaccine is instantaneous, and we therefore have in these three tubes, something comparable to thirty patients, of whom the first 10 have a diminishingly severe staphylococcal infection, the second 10 a similar series of infections, but also a small dose of tuberculin and the third 10 a larger dose. What happened? By a technique which need not be detailed, Wright was able to find out the number of organisms which survived in each separate cubic millimetre of blood and the result was broadly as follows. In the case of the heavily implanted bloods the eftect of the large dose of vaccine was to allow them to grow more than twice as many colonies as in the control blood, whereas the small dose of vaccine reduced the number to nearly half what grew in the controls. In the lightly implanted bloods, however, the large dose of vaccine enhances the bactericidal power more than does the small dose. From this and similar experiments, Wright deduces that there is a certain dose of antigen, which produces the optimum immunity response. If the blood is already heavily infected it requires only a small addition of antigen to bring the concentration up to the optimum, but if the infection is slight then more of the artificial antigen is required. The importance of determining the correct dose is therefore obvious, and Wright has devised "the vaccine response test" (Lancet, March 10, 1923) to find out what is the optimum amount of vaccine to give. The result of this and similar experiments is such as to make Wright say in a recent publication in the Lancet (August 15, 1931), "That when I operate on my blood with a tubercle vaccine (B.E.) I get better-that is, more emphatic, and for the same dose employed, more consistent-immunising response than when I operate with staphylococcus vaccine." For this reason he recommends that when the nature of the infecting agent cannot be determined, "a passionate conviction that immunity is specific should not be allowed to hold us back from inoculating minimal doses of tuberculin B.E. or in the cases associated with rheumatoid changes, a vaccine of intestinal streptococci."

With regard to the variety of tuberculin employed, the nearer we get to nature, the better, so up to date I have always used the bacillary emulsion.

The theoretical rationale for this is as follows:-For the most part, antigens are proteins. It is not known yet to what they owe their antigenic properties, though there is evidence that the benzene rings play an important part. Thus gelatine which contains no tyrosine or tryptophane is devoid of antigenic properties while other proteins possessing a full complement of aromatic radicles but deficient in some of the amino-acids commonly. present, possess definite antigenic properties. 
Another point about antigens is that they must be non-diftusible colloids. The reason for this is that if they could enter the cells, they would be destroyed therein, but as the cell cannot deal with them in this way it must elaborate some extracellular antibody to react with the foreign protein as a prelude to its destruction. Evidence of this is afforded by the observation that the cleavage products of an antigen even when injected all together have no antigenic capacity, but if reunited into colloidal molecules this capacity is restored. In therapeutics, we are of course concerned with the production of antibodies, and we have already seen that the amount of these which is produced does not vary directly with the amount of antigen injected. In fact small doses often produce more than large doses, and this is a point which is of great importance in determining the dosage of tuberculin.

In this connection one has also to consider the question of allergy. One cannot help feeling that some of the chronic eye diseases are of this nature, though I admit it has not been proved except in the case of interstitial keratitis and phlyctenular disease, and one knows that the whole secret of desensitisation is to give very small doses. My practice, therefore, is to start with something like $1 / 500,000 \mathrm{mg}$. of B.E. and work the dose up very slowly. Rich and McCordock have some interesting things to say on this subject and draw a distinction between the immunity and allergic reactions. The immunity reaction leads to death of the bacilli while the allergic reaction is the response of the tissues to the liberated protein. By administering repeated, increasing doses of tuberculin to the allergic human subject, a desensitisation to tuberculin may be established to such a degree that the individual becomes able to tolerate relatively enormous doses, without inflammation, necrosis, or constitutional symptoms. Tissue necrosis in tuberculosis is almost entirely the result of allergic hypersensitiveness to tuberculo-protein and if resistance is independent of allergy, the reduction of the necroticising effect of hypersensitiveness to the bacillary proteins could easily be understood to be beneficial. The formation of tubercles would not appear to be an allergic reaction, since it can be brought about by a lipoid extracted from the bacillus which lipoid itself does not produce any allergic changes. For the production of allergy it is necessary to use whole bacilli, and it appears that it has not yet been possible to extract from them the true sensitising antigen. By analogy, therefore, desensitisation would appear to be best produced by the same reagent, i.e., whole bacilli and not extracts, and this seems to furnish an additional indication for the use of tuberculin B.E. in preference to T.R., etc. Another way of looking at allergy is to regard it as a change in the body whereby the relatively bland products of the breakdown of tubercle bacilli are converted into 
powerful irritants and poisons without any apparent gain to the organism in the way of immunity. In fact, allergic reactions have the effect of spreading rather than fixing the bacilli. Thus Krause and Willis produced allergic reactions with tuberculin, in the skin of immunised animals, and into these inflamed areas injected virulent bacilli. These animals developed more widely spread tuberculosis than did controls where the injection was made into normal areas of skin. Healing of tuberculous foci is also thought to be interfered with by allergy.

This separation of immunity and allergy seems important and would explain very nicely some of the mild cases of doubtful tuberculous iritis. An old focus of tuberculosis, for example, lights up as a result of extreme exhaustion; the tubercle bacilli are disintegrated as the patient has acquired immunity and in their disintegration they set free tuberculo-protein. Unfortunately the patient is also allergic and therefore develops the so-called tuberculids such as asthma, iritis, or arthritis.

The bad effect of allergy is shown very strikingly by some tissue culture experiments performed by Rich and Lewis (Proc. Soc. Exp. Biol. and Med., Vol. XXV, p. 596, 1928).

They placed thoroughly washed, fixed tissue and blood cells of allergic and of normal guinea pigs in the plasma of normal and of allergic animals, and the same amount of tuberculin was added to all the cultures. The cells from allergic animals were regularly and promptly killed when exposed to tuberculin, regardless of the plasma in which they were placed, while the cells from normal animals, regardless of the plasma in which they were placed, remained alive and multiplied when exposed to the same concentrations of tuberculin which rapidly killed the allergic cells.

Turning to the clinical side, before discussing purely ophthalmic cases, I should like to allude to some experiences of Houghton in a tuberculosis sanatorium. His results are not ready yet for publication, but he was kind enough to write to me a very interesting letter which seems to have a strong bearing on the non-specific effect of tuberculin. He says:- "I have been for some time convinced that infection with tuberculosis (by which I mean active disease) confers a considerable degree of resistance to infection by other organisms, particularly streptococcal and pneumococcal and to some extent staphylococcal infection. This is, however, an extremely difficult contention to prove. The idea of any non-specific immunity suggested itself to me as the result of my own clinical experience of tuberculosis during the past four years, during which time I have seen no acute infections attributable to the common organisms except in cases in which the diagnosis of tuberculosis has not been confirmed. I have suggested this to others whose experience of tuberculosis is far greater than mine and they have, on consideration, 
agreed. So far this is a purely clinical opinion. The hospital has 350 beds devoted entirely to pulmonary tuberculous cases of all ages, and on examining the statistics for the past ten years, I find that absence of non-tuberculous disease among the patients is striking. I am at present engaged in going into the case papers in detail, but am not yet in a position to give any actual figures. In any case these figures would be of little statistical value without a set of figures for comparison, and that, of course, is one's great difficulty. I have, in the past, attempted animal experiments, but they are intended rather as preliminary efforts. Encouraged however, by Sir Almroth Wright's work, I am now carrying on with these on a larger scale. I think it is a common experience to find that in cases of tuberculous empyema which have had a rib resected for drainage purposes, and which do eventually become secondarily infected, the activity of the T.B. process subsides, and a chronic discharging sinus results. I have treated one or two such cases as these with tuberculin, on the principle that the secondary infection has overcome the T.B. infection. The results have been very encouraging, the pus becoming scantier and the sinus tending to close. I am also attempting to raise the resistance to T.B. infection by the converse process, but although I have a considerable number of cases under observation, I am not yet in a position to give an opinion. I have not yet published anything on this question as I feel that at present my ideas are too indefinite. I am hoping, however, within the next few months to have some more concrete facts to produce."

These statements seem to bear out Wright's experimental observations, and afford some justification for the ophthalmic surgeon resorting to tuberculin in cases of obscure aetiology.

The following cases may be of interest from the clinical standpoint:-

The first was a woman, E.H., aged 37 years, whom I took into hospital for disciform keratitis in the left eye. All investigations were negative, except that dental sepsis was present, and five infected teeth were removed. On leaving the wards, she had fine keratic precipitates, a partially healed focus of choroido-retinitis, and a considerable amount of vitreous haze. She had her first dose of tuberculin on January 4, 1930, and when I saw her on January 10, she asserted that she felt very much better, though the haze was still very thick. A fortnight later it was beginning to clear, and by the end of March she could see 6/12. In July, i.e., six months after starting tuberculin, vision was $6 / 5$ partly, and the haze was almost gone, except for a ring-like opacity in the vitreous, which resembled the condition seen in a case of Sir William Lister's, and described as a hole in the hyaloid. The tuberculin was therefore stopped; she was by now up to $1 / 50 \mathrm{mg}$., but she was instructed to return for a 
further course of injections in two months. The patient waited for nearly four months before coming back. By this time, vision had gone down to 6/12 partly, and the vitreous haze had returned. Tuberculin administration was resumed, and within five weeks the haze had almost disappeared, with a corresponding improvement of vision up to $6 / 5$ partly. The last occasion on which I saw her was in March of this year, when vision was 6/6 partly. An obvious criticism of this case is that it was probably one of tuberculous keratitis and choroiditis, but all I can say is that there was no clinical evidence of tuberculosis, and that the precipitates were at no time of the mutton-fat type. This of course does not exclude tuberculosis as an aetiological factor, but whatever one may think about the choroid, one would not expect a tuberculous keratitis to clear up in this way, and leave no sequelae of vascularisation, etc.

The beneficial effects of tuberculin in scleritis and episcleritis are too well known to need much description, but the following case may be of interest, if only by way of supporting Wright's theory about the optimum dose of antigen. M.C., a woman, 45 years of age, consulted me at home in September, 1924, with a bad attack of scleritis in the right eye, the left one having been enucleated some 30 years before. I sent her to the Central London Ophthalmic Hospital with a note to the house surgeon, advising an injection of $1 / 200,000 \mathrm{mg}$. of tuberculin. Two days later, when I saw her at hospital, I was surprised to find that the eye was white, and free of all evidences of active inflammation. It was rather a dramatic change, and I asked the house surgeon what dose she had given of tuberculin, and received the reply, $1 / 20,000 \mathrm{mg}$. I should never have dared to start with a dose like that myself, but the result was satisfactory. This patient has been under observation at intervals since this time, and has had a few relapses, each of which has been cured by tuberculin. I saw her last three weeks ago with a perfectly white eye for the last seven months, during the whole of which time she had been on tuberculin B.E.

A case of another type is H.C., who suffered from attacks of recurrent iritis for something like 30 or 40 years. She had every sort of treatment one could think of, and was the type of recurrent iritis which is associated with rheumatoid changes. It was not until December, 1929, that I thought of trying tuberculin, and then largely because there seemed to be some episcleritis associated with her attacks of iritis. As long as she continued her injections, she remained free of attacks, but if she stopped them for only a short period, she seemed to get a relapse. Last November she had hemiplegia, and died shortly after from heart failure.

Another case in which tuberculin had a remarkable remedial effect was that of Miss W., who had numerous small staining areas on her cornea, with much photophobia and lacrymation. The usual local 
treatment produced no effect, nor did the removal of some infected teeth, in fact the condition became worse, and after some days of in-patient treatment, I noted the development of a deep infiltrate in the cornea of a disciform type. Remembering the first case, I thought it would be worth while to try tuberculin again, and the result was that within a few days the deep opacity had disappeared, and the superficial areas were beginning to heal.

Another case of chronic iritis may have been definitely tuberculous. She was a woman aged 49 years, who had had recurrent iritis since the age of 17 , and was under the care of Mr. Paton. When he saw her in July of last year, both irides were almost completely bound down, and there was a history of a past attack of iritis with raised tension and pain. The patient had to return to India in a few months, so a double iridectomy was indicated. No septic focus was found, so she was given milk injections and intensive salicylate treatment with little effect. I saw her several times during $\mathrm{Mr}$. Paton's absence on holiday, and ordered tuberculin. The first dose of $1 / 100,000 \mathrm{mg}$. produced no effect, but the second of $1 / 75,000$ produced a little shivering, and some pain in the eye. The dose was therefore reduced, and then worked up more slowly, with a result that in a month the condition of the eye was markedly improved, and iridectomy was performed two weeks later without any complications.

I am afraid that these case reports are thoroughly empirical and unscientific, but it is difficult to know quite where to begin if the subject is to be worked out satisfactorily.

It seems to me that we have two questions to decide.

1. Are we missing a certain number of cases of tuberculosis by falsely attributing the lesion in the eye to some other cause, and not finding the true diagnosis until tuberculin is administered?

2. Has tuberculin a definitely non-specific immunising effect against organisms other than the tubercle bacillus and thus brings about cure?

With regard to the first question, how are we to exclude a tuberculous aetiology? We know that tuberculous affections of the cornea and uveal tract do not by any means always give rise to pathognomonic changes, and, we have the assurance from pathologists that something like 90 per cent. of human beings harbour a tuberculous infection somewhere in their bodies.

This is emphasized particularly by Urbanek. In an examination of 500 cases of tuberculous uveitis, he pointed out that there was no typical clinical or pathological picture that indicated a tuberculous involvement of the eye, and this even with the slit-lamp examination. He goes so far as to say that acute tuberculous conditions may even present the well-known appearance of gonococcal iritis, with its gelatinous aqueous. 
With regard to the second question, we have Sir Almroth Wright's work, which seems to show that tuberculin B.E. is a very good antigen for staphylococcal infections. I feel, therefore, that the therapeutic, non-specific effect of tuberculin is a matter that ought to be subjected to further scientific investigation.

\title{
REFERENCES
}

1. Wright, Sir Almroth.-Lancet, August 1, 8 and 15, 1931.

2. Lancet, March 10, 1923.

3. Rich and McCordock.-Bull. Johns Hopkins Hosp., No. 5, Vol. XLIV, May, 1929.

4. Rich and Lewis.-Proc. Soc. Exp. Biol. and Med., Vol. XXV, p. 596, 1928.

\section{HARDNESS OF THE EYE: AN HISTORICAL NOTE}

\author{
BY \\ ARNOLD SORSBY
}

LONDON

WHEN Brisseau ${ }^{1}$ established, in 1709 , that cataract was a change in the lens itself, and not an organised opacity in front of it, he also advanced the view that glaucoma, which up to then was regarded as dessication of the lens, was not a lesion of the lens at all, but a change in the vitreous. The clinical conception of glaucoma then prevailing was no less confused, and 150 years had to pass before the essential features of the disease were clearly recognised. The general recognition of such a thing as hardness of the eye had still to come, and its recognition as the characteristic sign of glaucoma was a very late achievement.

William Mackenzie ${ }^{2}$ is generally credited with establishing hardness as a feature of glaucoma. In his classical textbook (1st edition, 1830) he repeatedly stresses this association. He contrasts the blindness of glaucoma with that of cataract:

The eyeball in glaucomatous amaurosis always feels firmer than natural; while in cataract, it presents its usual degree of resistance to the pressure of the finger. (1st edition, p. 572.)

On the succeeding page (p. 573), he states, in discussing the cataractous eye :

A stony hardness of the eye denotes glaucoma.

$\mathrm{He}$ also recognised acute glaucoma, though this term is not employed in the first edition, making its first appearance in the 